\title{
A Forgetting-based Approach to Merging Knowledge Bases
}

\author{
Dai Xu, Xiaowang Zhang and Zuoquan Lin \\ Department of Information Science, Peking University \\ Beijing 100871, P. R. China \\ Email: $\{$ xudai,zxw,lzq $\} @$ is.pku.edu.cn
}

\begin{abstract}
This paper presents a novel approach based on variable forgetting, which is a useful tool in resolving contradictory by filtering some given variables, to merging multiple knowledge bases. This paper first builds a relationship between belief merging and variable forgetting by using dilation. Variable forgetting is applied to capture belief merging operation. Finally, some new merging operators are developed by modifying candidate variables to amend the shortage of traditional merging operators. Different from model selection of traditional merging operators, as an alternative approach, variable selection in those new operators could provide intuitive information about an atom variable among whole knowledge bases.

Keywords-belief merging; variable forgetting; dilation; inconsistency handling; knowledge base
\end{abstract}

\section{INTRODUCTION}

The knowledge bases (KBs) contain a large amount of information coming from different sources. KBs must be able to "intelligently" manage such distributed information. An important task is ensuring that those $\mathrm{KBs}$, comprising collections of information which possibly conflict with each other, need to be combined into a consistent whole ([1]). As one of traditional ways of management, belief merging concerns with the problem of determining a group's beliefs from individual members' beliefs ([2], [3]). The merging process from the point of logic is formalize as follows: let $\Phi=\left\{\varphi_{1}, \cdots, \varphi_{n}\right\}$ be a group of $\mathrm{KBs}$ (which is composed of multiple formulae and can also be taken as conjunction of them from logic equivalence) where each logical formula $\varphi_{i}$ denotes a knowledge base $(\mathrm{KB})$ from some information source. $\Phi$ is a multi-set of formulae which is to merge. $\mu$ is a set of formulae which represents the integrity constraints, i.e., some information that the result of merging must obey. The goal of merging is to obtain a new $\mathrm{KB} \triangle_{\mu}(\Phi)$ which represents the consensus of $n$ sources given integrity constraints $\mu$ ([4]).

When conflicts occur in $\mathrm{KBs}$, we intuitively weaken them to achieve consistency again. Thus we can avoid the trivialization of inference that everything can be deducted from inconsistent $\mathrm{KBs}$. Of course, a fundamental requirement is to minimize changes of the original KBs in maintaining their consistency. That is, the information of the original KBs should be preserved as much as possible. Those current approaches are based on two fundamentally different standpoints: the syntaxbased and the semantics-based. Based on syntax, the maximal consistent subsets of the original bases consistent with the integrity are selected as the merged result. Unfortunately, the common weakness is that the merged results depend on the syntax forms of KBs. Based on semantics (models), those models which have the minimal distance to models of the KBs are selected from the models of integrity constraints as the candidates models of the merged result. The Dalal distance has been proved a useful way to characterize two models $([5])$. Based on three methods of aggregating distances of multi-KBs, there are three merging operators, namely, $\triangle_{\mu}^{\Sigma}, \triangle_{\mu}^{M a x}$ and $\triangle_{\mu}^{G M a x} . \triangle_{\mu}^{\Sigma}$ is taking the summation of distances as the aggregation to pick out the most popular models $([6]) . \triangle_{\mu}^{M a x}$ is taking the maximum of distances as the aggregation to minimize the worst cases ([7]). $\triangle_{\mu}^{G M a x}$ is based on lexicographical order to capture the arbitration behavior of operator $\triangle_{\mu}^{\operatorname{Max}}([2])$.

However, three existing merging operators do not always work well in some cases. We still consider an example of swimming-pool discussed in [2] previously.

Swimming-pool At a meeting of a block of flat co-owners, the chairman proposes for the coming year the construction of a swimming-pool, of a tennis-court and of a private-car-park. But if two of these three items are built, the rent will significantly increase. We will denote by $S, T, P$ respectively the construction of the swimming-pool, the tennis-court and the private-car-park. We denote $I$ the rent increase. The chairman outlines that building two items or more will have an important impact on the rent: $\mu=((S \wedge T) \vee(S \wedge P) \vee(T \wedge P)) \rightarrow I$.

There are four co-owners $\Phi=\left\{\varphi_{1}, \varphi_{2}, \varphi_{3}, \varphi_{4}\right\}$. Two of the co-owners want to build the three items and do not care about the rent increase: $\varphi_{1}=\varphi_{2}=S \wedge T \wedge P$. The third one thinks that building an item will cause at some time an increase of the rent and wants to pay the lowest rent, So he is opposed to any construction: $\varphi_{3}=\neg S \wedge \neg T \wedge \neg P \wedge \neg I$. The last one thinks that the block really needs a tennis-court and a private-car-park but does not want a high rent increase: $\varphi_{4}=T \wedge P \wedge \neg I$. In [2], the merging results of applying three operators above are in the following.

$\triangle_{\mu}^{\Sigma}(\Phi)=S \wedge T \wedge P \wedge I$.

$\triangle_{\mu}^{M a x}=(\neg S \wedge \neg T \wedge P) \vee(\neg S \wedge T \neg P) \vee(S \wedge \neg T \wedge P \wedge I)$. $\triangle_{\mu}^{G M a x}=\neg S \wedge \neg I \wedge((\neg T \wedge P) \vee(T \wedge \neg P))$.

However, the results are not intuitive from the view of propositional symbols. For variable $I$, since $\varphi_{1}, \varphi_{2}$ don't care about it and $\varphi_{3}, \varphi_{4}$ support $\neg I$, So the literal formula $\neg I$ can be regarded as the perspective of the whole group. For variables $S, T, P$, the former two owners and the third one 
have different opinions. That is, the former two support $S, T$ and $P$ and the third one supports their opposite. So they don't reach consensus if no co-owner gives in. The formula $\neg I \wedge \mu$ is thus natural result for the merging. Moreover, $\triangle_{\mu}^{\Sigma}(\Phi)$ and $\triangle_{\mu}^{M a x}$ are not enough to capture $\neg I$. on the other hand, although $\triangle_{\mu}^{\text {GMax }} \models \neg I$, the operator is too strong since $\triangle_{\mu}^{\text {GMax }} \models \neg S$. Two co-owners support $S$, one supports its opposite and one is neutral about $S$. We can't agree on $\neg S$ even if we don't follow the majority property. It is reasonable that $S$ is taken as unknown, i.e., neither $S$ nor $\neg S$.

In general, inconsistencies occurring between KBs are caused by over-defining in representing something. The main idea of handling inconsistency is removing or ignoring those redundant information. As a significant approach to dealing with inconsistencies, forgetting is a useful tool to restrict variables to be discussed in a subset of variables of the original KBs with keeping logical equivalence locally ([י]).

In this paper, inspiring from [10], we present a novel approach based on variable forgetting to merging multi-KBs with maintaining consistency. First, we build the relationship between belief merging and variable forgetting by using the framework of dilation (presented in [3]). We then reformalize three classical merging operators via variable forgetting. Two new forgetting-based merging operators are obtained by modifying variables to be forgotten. We show that those forgettingbased merging operators can amend the shortage (discussed in the motivating example of "swimming-pool") of classical operators.

The rest of this paper is organized as follows. Section 2 gives a brief review of merging and forgetting for KBs. Section 3 employs forgetting to capture traditional merging operators. We develop three new merging operators by forgetting variables in Section 4. in the last section, we summarize this paper and put forward to some future works.

\section{PReliminaries}

In this section, we briefly review basic concepts of belief merging and forgetting for propositional KBs.

\section{A. Belief Merging}

The propositional language, denoted by $\mathcal{L}$, is constructed from a finite set $\mathcal{P}$ of symbols. $T$ (true) and $\perp$ (false) are boolean constants. In $\mathcal{L}, p$ denotes a propositional variable, $\varphi, \psi, \mu$ propositional formula, $\Phi, \Phi_{1}, \Phi_{2}, \cdots$ sets of formulas. $\Phi_{1} \cup \Phi_{2}$ is the union of sets $\Phi_{1}, \Phi_{2}$, and $\Phi_{1} \sqcup \Phi_{2}$ is the union of multi-sets of sets $\Phi_{1}, \Phi_{2} . \operatorname{Var}(\varphi)$ denotes the set of variables which occur in $\varphi$. An interpretation is a function from $\mathcal{P}$ to $\{0,1\} . \omega, \omega^{\prime}$ denote interpretations. $\mathcal{M}$ is the collection of all interpretations. An interpretation is a model of formula iff it makes it true in classical way. $\bmod (\varphi)$ denotes the set of models of $\varphi . \varphi$ is consistent iff $\bmod (\varphi) \neq \emptyset . \varphi \equiv \psi$ iff $\bmod (\varphi)=\bmod (\psi)$.

The aggregation of finite KBs into a collective one is studied by a recent discipline called belief merging (see [8], [2], [3]). A particular type of aggregation is called model-based merging. Intuitively, the model-based merging is aggregating those models which are closer to models of every formula. In technique, it is choosing those models which have the minimal aggregating distance.

Let $\Phi^{\prime}=\left\{\varphi_{1}^{\prime}, \cdots, \varphi_{n}^{\prime}\right\}, \Phi \leftrightarrow \Phi^{\prime}$ iff there is a bijection $f$ : $\Phi \rightarrow \Phi^{\prime}$ satisfying $\forall \varphi_{i} \in \Phi, \varphi_{i} \leftrightarrow f\left(\varphi_{i}\right)$.

$|V|$ denotes the cardinal number of a set $V$. The (Dalal) distance between two models $d\left(\omega, \omega^{\prime}\right)=\mid\left\{p \mid \omega, \omega^{\prime}\right.$ assign differently on $p\} \mid$. The distance between a model and formula is defined as $d(\omega, \varphi)=\min _{\omega^{\prime} \in \bmod (\varphi)} d\left(\omega, \omega^{\prime}\right)$.

$\varphi_{x \leftarrow 0}\left(\right.$ resp. $\left.\varphi_{x \leftarrow 1}\right)$ denotes the formula obtained by replacing in $\varphi$ every occurrence of variables $x$ by $\perp$ (resp. $\top$ ).

Let $\Phi=\left\{\varphi_{1}, \cdots, \varphi_{n}\right\}, \wedge \Phi=\wedge_{\varphi_{i} \in \Phi} \varphi_{i}$. Formulas $\varphi, \varphi^{\prime}$ denote KBs, $\mu, \mu_{1}, \mu_{2}$ represent the integrity constraints. For simple discussion, we continue to assume every formula $\varphi_{i} \in$ $\Phi$ is consistent in this paper.

There are nine postulates (IC0)-(IC8) presented in [2] to capture the belief merging.

Let $\triangle$ be an IC merging operator iff it satisfies the following postulates:

(IC0) $\triangle_{\mu}(\Phi) \vdash \mu$

(IC1) If $\mu$ is consistent, then $\triangle_{\mu}(\Phi)$ is consistent

(IC2) If $\wedge \Phi$ is consistent with $\mu$, then $\triangle_{\mu}(\Phi)=\wedge \Phi \wedge \mu$

(IC3) If $\Phi_{1} \leftrightarrow \Phi_{2}$ and $\mu_{1} \leftrightarrow \mu_{2}$, then $\triangle_{\mu_{1}}\left(\Phi_{1}\right) \leftrightarrow \triangle_{\mu_{2}}\left(\Phi_{2}\right)$

(IC4) If $\varphi \vdash \mu$ and $\varphi^{\prime} \vdash \mu$, then $\triangle_{\mu}\left(\varphi \sqcup \varphi^{\prime}\right) \wedge \varphi \forall \perp \Rightarrow$ $\triangle_{\mu}\left(\varphi \sqcup \varphi^{\prime}\right) \wedge \varphi^{\prime} \not \perp$

(IC5) $\triangle_{\mu}\left(\Phi_{1}\right) \wedge \triangle_{\mu}\left(\Phi_{2}\right) \vdash \triangle_{\mu}\left(\Phi_{1} \sqcup \Phi_{2}\right)$

(IC6) If $\triangle_{\mu}^{\Phi_{1}} \wedge \triangle_{\mu}\left(\Phi_{2}\right)$ is consistent, then $\triangle_{\mu}\left(\Phi_{1} \sqcup \Phi_{2}\right) \vdash$ $\triangle_{\mu}\left(\Phi_{1}\right) \wedge \triangle_{\mu}\left(\Phi_{2}\right)$

(IC7) $\triangle_{\mu_{1}}(\Phi) \wedge \mu_{2} \vdash \triangle_{\mu_{1} \wedge \mu_{2}}(\Phi)$

(IC8) if $\triangle_{\mu_{1}}(\Phi) \wedge \mu_{2}$ is consistent, then $\triangle_{\mu_{1} \wedge \mu_{2}}(\Phi) \vdash$ $\triangle_{\mu_{1}}(\Phi) \wedge \mu_{2}$

Besides (IC0)-(IC8), there are some additional postulates to characterize the other properties as follows:

The majority property (Maj): $\exists n \triangle_{\mu}\left(\Phi_{1} \sqcup \Phi_{2}^{n}\right) \vdash \triangle_{\mu}\left(\Phi_{2}\right)$. Intuitively, if a subgroup appears enough in the whole group, then it is the opinion of the group.

Majority independence requires us only consider different KBs. It is denoted by (MI): $\triangle_{\mu}\left(\Phi_{1} \sqcup \Phi_{2}^{n}\right) \leftrightarrow \triangle_{\mu}\left(\Phi_{1} \sqcup \Phi_{2}\right)$.

There are three traditional model-based merging operators as follows: $\triangle_{\mu}^{\Sigma}$ (presented in [6]), $\triangle_{\mu}^{\operatorname{Max}}$ (presented in [7]) and $\triangle_{\mu}^{G M a x}$ (presented in [2]).

Let $\Phi$ be a KB and $\omega, \omega^{\prime}$ two interpretations.

- The $\Sigma$-distance between an interpretation and a KB is defined as $d_{\Sigma}(\omega, \Phi)=\Sigma_{\varphi \in \Phi} d(\omega, \varphi)$. Then we have the following pre-order: $\omega \leq_{\Phi}^{\Sigma} \omega^{\prime}$ iff $d_{\Sigma}(\omega, \Phi) \leq d_{\Sigma}\left(\omega^{\prime}, \Phi\right)$. The merging operator $\triangle_{\mu}^{\Sigma}$ is defined: $\bmod \left(\triangle_{\mu}^{\Sigma}\right)=$ $\min \left(\bmod (\mu), \leq_{\Phi}^{\Sigma}\right)$.

It easily shows that $\triangle_{\mu}^{\Sigma}$ satisfies (IC0)-(IC8), (Maj).

- The Max-distance between an interpretation and a KB is defined as follows: $d_{\operatorname{Max}}(\omega, \Phi)=\operatorname{Max}_{\varphi \in \Phi} d(\omega, \varphi)$. Then we have the following pre-order: $\omega \leq_{\Phi}^{\operatorname{Max}} \omega^{\prime}$ iff $d_{\operatorname{Max}}(\omega, \Phi) \leq d_{\text {Max }}\left(\omega^{\prime}, \Phi\right)$. The merging operator $\triangle_{\mu}^{\operatorname{Max}}$ is defined as follows: $\bmod \left(\triangle_{\mu}^{\operatorname{Max}}\right)=$ $\min \left(\bmod (\mu), \leq_{\Phi}^{\text {Max }}\right)$.

It easily shows that $\triangle_{\mu}^{\operatorname{Max}}$ satisfies (IC0)-(IC5), (IC7), (IC8) and (MI). In particular, it can't satisfy (IC6), 
(Maj)

- Suppose $\Phi=\left\{\varphi_{1}, \cdots, \varphi_{n}\right\} \cdot d_{j}^{\omega}=d\left(\omega, \varphi_{j}\right)$. Let $L_{\omega}^{\Phi}$ be the list obtained from $\left(d_{1}^{\omega}, \cdots, d_{n}^{\omega}\right)$ by sorting it in descending order. Let $\leq_{l e x}$ be the lexicographical order between sequences of integers. Then the pre-order $\leq_{\Phi}^{G M a x}$ is defined as follows: $\omega \leq_{\Phi}^{G M a x} \omega^{\prime}$ iff $L_{\omega}^{\Phi} \leq_{l e x}$ $L_{\omega^{\prime}}^{\Phi}$. The merging operator $\triangle_{\mu}^{G M a x}$ is defined as follows: $\bmod \left(\triangle_{\mu}^{G M a x}=\min \left(\bmod (\mu), \leq_{\Phi}^{G M a x}\right)\right.$.

It easily shows that $\triangle_{\mu}^{G M a x}$ satisfies (IC0)-(IC8).

Some other merging operators such as $\mathrm{DA}^{2}$ presented in [4] could be taken as extensions of three classical operators.

\section{B. Forgetting}

Forgetting proposed by Lin and Reiter ([9]) is filtering all facts that are no longer true from KBs. That is to say, forgetting is taken as a basic operation for weakening formulas. In this sense, variables forgetting could be employed to reason under inconsistency $([10])$.

Let $\varphi$ be a propositional formula, $p$ be an atom and $V$ be a set of variables. $\exists V . \varphi$ denotes forgetting $V$ in $\varphi$ which is recursively defined as follows:

- $\exists \emptyset . \varphi \equiv \varphi$;

- $\exists\{p\} \cdot \varphi \equiv \varphi_{p \leftarrow 0} \vee \varphi_{p \leftarrow 1}$;

- $\exists(V \cup\{p\}) \cdot \varphi \equiv \exists V .(\exists\{p\} \cdot \varphi)$.

Let $\operatorname{switch}(\omega, p)$ denote the interpretation that assigns the same truth values to all variables except $p$, and assigns the opposite value to $p$. Then

$\bmod (\exists\{p\} \cdot \varphi)=\bmod (\varphi) \cup\{\operatorname{switch}(\omega, p) \mid \omega \models \varphi\}$

Next we enumerate some good properties of forgetting for knowledge bases which will be useful for our work.

- Let $\varphi$ be consistent, $V_{1} \subseteq V_{2} \subseteq \operatorname{Var}(\varphi)$, then $\exists V_{1} . \varphi \models$ $\exists V_{2} . \varphi$. In particular $\varphi \models \exists V . \varphi$.

- $V=\operatorname{Var}(\varphi)$. If $\varphi$ is consistent, then $\exists V \cdot \varphi \models \top$; If $\varphi$ isn't consistent, then $\exists V . \varphi \models \perp$.

- Let $\varphi$ be consistent, if $\operatorname{Var}(\varphi) \subseteq V$, then $\exists V \cdot \varphi \models \top$.

- Let $\varphi, \psi$ be two formulas, $V$ be a variables set, then $\exists V .(\varphi \vee \psi) \equiv \exists V . \varphi \vee \exists V . \psi$.

- If $p \notin \operatorname{Var}(\varphi)$, then $\exists\{p\} . \varphi \equiv \varphi$.

- If $\varphi \models \varphi^{\prime}$ then $\exists V . \varphi \models \exists V . \varphi^{\prime}$. In particular, if $\operatorname{Var}\left(\varphi^{\prime}\right) \cap$ $V=\emptyset$ then $\exists V \cdot \varphi \models \varphi^{\prime}$.

\section{RElationship BetweEn MERGING AND FORGETTING}

In this section, we apply variable forgetting to capture three existing merging operators $\triangle_{\mu}^{\Sigma}, \triangle_{\mu}^{M a x}, \triangle_{\mu}^{G M a x}$. At first, we need introduce a so-called operator of dilation (presented in [3]) to build the inner relationship between belief merging and variable forgetting.

A dilation operator $D$ is a mapping from formula to formula satisfying: $\bmod (D(\varphi))=\{\omega \in \mathcal{M} \mid d(\omega, \varphi) \leq 1\}$.

We have $D^{1}(\varphi)=D(\varphi)$ and $D^{n}(\varphi)=D\left(D^{n-1}(\varphi)\right)$. So $\bmod \left(D^{n}(\varphi)\right)=\{\omega \in \mathcal{M} \mid d(\omega, \varphi) \leq n\}$.

Let $\Phi=\left\{\varphi_{1}, \varphi_{2}, \cdots, \varphi_{n}\right\}$. These operators can be equivalently expressed by dilation.
- $\triangle_{\mu}^{\Sigma}(\Phi) \equiv \vee_{c_{1}+\cdots+c_{n}=k}\left(D^{c_{1}}\left(\varphi_{1}\right) \wedge \cdots \wedge D^{c_{n}}\left(\varphi_{n}\right) \wedge \mu\right)$, where $k$ is the least number so that the disjunction is consistent.

- $\triangle_{\mu}^{\operatorname{Max}}(\Phi) \equiv D^{k}\left(\varphi_{1}\right) \wedge \cdots \wedge D^{k}\left(\varphi_{n}\right) \wedge \mu$, where $k$ is the least number so that $D^{k}\left(\varphi_{1}\right) \wedge \cdots \wedge D^{k}\left(\varphi_{n}\right) \wedge \mu$ is consistent.

- $\triangle_{\mu}^{G M a x}(\Phi) \equiv \vee_{<c_{1}, \cdots, c_{n}>\in \operatorname{perm}(T)}\left(D^{c_{1}}\left(\varphi_{1}\right) \wedge \cdots \wedge\right.$ $\left.D^{c_{n}}\left(\varphi_{n}\right) \wedge \mu\right)$, where $T$ is an n-tuple of integers, which is sorted in descending order, is lexicographically least such that the disjunction is consistent.

Next we present that dilation can be captured by forgetting. Before presenting, a key lemma will be stated in the following.

Lemma 1: Let $\varphi$ be a formula and $V$ be a set of variables. We have $\forall \omega \in \bmod (\exists V . \varphi), d(\omega, \varphi) \leq|V|$.

Proof: $\forall \omega \in \bmod (\exists V . \varphi)$, there must be $\omega^{\prime} \in \bmod (\varphi)$ which satisfies $\omega, \omega^{\prime}$ assume the same on variables except for variables of $V$. So $d\left(\omega, \omega^{\prime}\right) \leq|V|$. Thus $d(\omega, \varphi) \leq|V|$.

Theorem 1: Let $\varphi$ be a consistent formula, $p$ be a propositional variable, $V$ be a set of variables and $n$ be an integer. We have

- $D(\varphi) \equiv \vee_{p \in \operatorname{Var}(\varphi)} \exists\{p\} \cdot \varphi$.

- If $1<n \leq|\operatorname{Var}(\varphi)|$, then $D^{n}(\varphi) \equiv$ $\vee_{V \subseteq \operatorname{Var}(\varphi),|V|=n} \exists V \cdot \varphi$.

- If $n>|\operatorname{Var}(\varphi)|$, then $D^{n}(\varphi) \equiv \top$.

Proof: 1. We will prove they have the same models. $\forall \omega \in \bmod (D(\varphi))$, then $d(\omega, \varphi) \leq 1$. If $d(\omega, \varphi)=0$ then $\omega \in \bmod (\varphi)$. So $\omega \in \bmod (\exists\{p\} . \varphi)$ and $\omega \in$ $\bmod \left(\vee_{p \in \operatorname{Var}(\varphi)} \exists\{p\} . \varphi\right)$. If $d(\omega, \varphi)=1$, then $\exists \omega^{\prime} \in \bmod (\varphi)$ and $d\left(\omega, \omega^{\prime}\right)=1$. So $\omega, \omega^{\prime}$ interpret the same except $p$ in $\operatorname{var}(\varphi)$. Thus $\omega \in \bmod (\exists\{p\} . \varphi)$. Otherwise, if $\omega \in$ $\bmod \left(\vee_{p \in \operatorname{Var}(\varphi)} \exists\{p\} . \varphi\right)$ then $\omega \in \bmod (\exists\{p\} . \varphi)$ for some $p$. So $d(\omega, \varphi) \leq 1$ holds by the lemma.

2. If $1<n \leq|\operatorname{Var}(\varphi)|$, Then $D^{n}(\varphi)=D\left(D^{n-1}(\varphi)\right)$. Suppose $D^{n-1}(\varphi)=\vee_{V \subseteq \operatorname{Var}(\varphi),|V|=n-1} \exists V . \varphi$. And $D^{n}(\varphi)=D\left(\vee_{V \subseteq \operatorname{Var}(\varphi),|V|=n-1} \exists V \cdot \varphi\right)$

$=\vee_{p \in \operatorname{Var}(\varphi)} \exists\{p\} .\left(\bigvee_{V \subseteq \operatorname{Var}(\varphi),|V|=n-1} \exists V . \varphi\right)$

$=\vee_{V \subseteq \operatorname{Var}(\varphi),|V|=n} \exists V . \varphi \vee \vee_{V \subseteq \operatorname{Var}(\varphi),|V|=n-1} \exists V \cdot \varphi$

$=\vee_{V \subseteq \operatorname{Var}(\varphi),|V|=n} \exists V \cdot \varphi$.

The last equation is because of a property of forgetting. Actually, $D^{n}(\varphi)=\vee_{V \subseteq \operatorname{Var}(\varphi),|V| \leq n} \operatorname{forget}(\varphi, V)$. And we can omit $V \in \operatorname{Var}(\varphi)$ in subscript without confusion, i.e., $D^{n}(\varphi)=\vee_{|V| \leq n} \operatorname{forget}(\varphi, V)$.

3. If $n>|\operatorname{Var}(\varphi)|$, then $\bmod \left(D^{n}(\varphi)\right)=\{\omega \in$ $\mathcal{M} \mid d(\omega, \varphi) \leq n\} . \exists \operatorname{Var}(\varphi) \cdot \varphi \equiv \top$, then $\forall \omega \in \mathcal{M}$, then $\omega \in \bmod (\exists \operatorname{Var}(\varphi) . \varphi)$. Thus $d(\omega, \varphi) \leq|\operatorname{Var}(\varphi)|<n$. So $\omega \in \bmod \left(D^{n}(\varphi)\right)$ and $D^{n}(\varphi)=\top$.

Since the relationship between dilation and forgetting is pointed out, it's natural to represent these operators by forgetting.

Theorem 2: Let $\Phi=\left\{\varphi_{1}, \varphi_{2}, \cdots, \varphi_{n}\right\}$.

- $\triangle_{\mu}^{\Sigma}(\Phi) \equiv \vee_{\left|V_{1}\right|+\cdots+\left|V_{n}\right|=k}\left(\exists V_{1} \cdot \varphi_{1} \wedge \cdots \wedge \exists V_{n} \cdot \varphi_{n} \wedge \mu\right)$.

- $\triangle_{\mu}^{\operatorname{Max}}(\Phi) \equiv \vee_{\left|V_{1}\right|=\cdots=\left|V_{n}\right|=k}\left(\exists V_{1} \cdot \varphi_{1} \wedge \cdots \wedge \exists V_{n} \cdot \varphi_{n} \wedge \mu\right)$.

- $\triangle_{\mu}^{G M a x}(\Phi)=\vee_{<\left|V_{1}\right|, \cdots,\left|V_{n}\right|>\in \operatorname{perm}(T)}\left(\exists V_{1} \cdot \varphi_{1} \wedge \cdots \wedge\right.$ $\left.\exists V_{n} \cdot \varphi_{n} \wedge \mu\right)$.

Here $k$ and $T$ are the same as above definitions. 
Proof: We only prove the first equality and the others are similar. Let $\triangle_{\mu}^{\Sigma_{\mu}}(\Phi) \equiv$ $\vee_{c_{1}+\cdots+c_{n}=k}\left(D^{c_{1}}\left(\varphi_{1}\right) \wedge \cdots \wedge D^{c_{n}}\left(\varphi_{n}\right) \wedge \mu\right)$ and $k$ is the least number that it is consistent. Then $\triangle_{\mu}^{\Sigma}(\Phi) \equiv$ $\vee_{c_{1}+\cdots+c_{n}=k}\left(\vee_{\left|V_{1}\right|=c_{1}}\left(\exists V_{1} \cdot \varphi_{1}\right) \wedge \cdots \wedge \vee_{\left|V_{n}\right|=c_{n}}\left(\exists V_{n} \cdot \varphi_{n}\right) \wedge\right.$ $\mu) \equiv \vee_{c_{1}+\cdots+c_{n}=k}\left(\bigvee_{\left|V_{1}\right|=c_{1}, \cdots,\left|V_{n}\right|=c_{n}}\left(\exists V_{1} \cdot \varphi_{1} \wedge \cdots \wedge\right.\right.$ $\left.\exists V_{n} \cdot \varphi_{n} \wedge \mu\right) \equiv \vee_{\left|V_{1}\right|+\cdots+\left|V_{n}\right|=k}\left(\exists V_{1} \cdot \varphi_{1} \wedge \cdots \wedge \exists V_{n} \cdot \varphi_{n} \wedge \mu\right)$.

A major difference between dilation and forgetting is that every variable has to be forgotten in dilation as it only find all the closest models to the original formula.

\section{Revised Merging Operators Using Forgetting}

The merging operators are represented by forgetting have the similar forms as by dilation. Since they have so close relationship that we can construct merging operators directly by forgetting. Our method is to revise the merging operators represented by forgetting to obtain some new ones which satisfy two new properties.

Theorem 2 shows that forgotten variables for different knowledge bases might be distinct. Thus the result of conjunction after forgetting doesn't focus on a special domain. If we restrict every $\mathrm{KB}$ to forget the same set of variables, we get a new operator defined in this domain. Of course, we consider the minimal sets of variables to make the result of KBs after forgetting consistent.

Literal formulas might be regarded as the simplest sublanguage which is constructed on a single atom. In the face of literal formula query, all other variables needn't to be considered. As the motivating example presented in Section 1, on the one hand, if some information sources entail a literal formula, but the others say nothing about this atom, then the literal formula should hold for the group because they don't conflict with each other about this atom. On the other hand, if some sources agree on a literal formula, but some sources object to it, then it should be rejected. So our attitude to merging is more skeptical than before based on this point.

Next we formalize such two properties.

(A1) Let a $\mathrm{KB} \Phi^{\prime} \subseteq \Phi$ and $l$ be a literal formula. Suppose $\mu \wedge l$ is consistent. If $\forall \varphi^{\prime} \in \Phi^{\prime}, \varphi^{\prime} \models l$ and $\operatorname{Var}(l) \notin \operatorname{Var}(\Phi-$ $\left.\Phi^{\prime}\right)$, then the merging result $\triangle_{\mu}(\Phi) \models l \wedge \mu$.

(A2) Let $\varphi_{1}, \varphi_{2} \in \Phi$. If $\varphi_{1} \models l$ and $\varphi_{2} \models \neg l$, then $\triangle_{\mu}(\Phi) \forall \forall l$. The property (A2) implicitly requires that $\triangle_{\mu}(\Phi) \not \forall \neg l$ holds too.

Though a $\mathrm{KB} \Phi$ is inconsistent w.r.t $\operatorname{Var}(\Phi)$, it may be consistent w.r.t some subset of $\operatorname{Var}(\Phi)$. We consider all these maximal subsets on set-inclusion or cardinal number and get two new operators in the following.

Definition 1: Let $\Phi=\left\{\varphi_{1}, \varphi_{2}, \cdots, \varphi_{n}\right\}$ and $\mu$ be an integrity constraint. $V \subseteq \operatorname{Var}(\Phi)$, the collection of minimal variables sets for forgetting $F S=\{V$ is minimal w.r.t set cardinal $\mid \wedge_{i=1}^{n} \exists V \cdot \varphi_{i} \wedge \mu$ consistent $\}$. We define $\triangle_{\mu}^{f_{1}}$ as follows: $\triangle_{\mu}^{f_{1}}(\Phi)=\vee_{V \in F S}\left(\wedge_{i=1}^{n} \exists V \cdot \varphi_{i} \wedge \mu\right)$.

The new operator satisfies (A1) and (A2). Before we prove the two properties above, we need the following lemma.
Lemma 2: Let $p$ be variable in $V, l$ be a literal formula on $p$. If $\forall 1 \leq i \leq n, \varphi_{i} \models l$, and $\wedge_{i=1}^{n} \exists V \cdot \varphi_{i}$ is consistent, then $\wedge_{i=1}^{n} \exists(V-\{p\}) \cdot \varphi_{i}$ is also consistent.

Proof: $\forall 1 \leq i \leq n, \varphi_{i} \models l$, so we obtain $\exists(V-$ $\{p\}) \cdot \varphi_{i} \models l$. Let $\omega \in \bmod \left(\wedge_{i=1}^{n} \exists V \cdot \varphi_{i}\right)$, then $\omega \in$ $\bmod \left(\exists V . \varphi_{i}\right)$. If $\omega \models l$ then $\omega \in \bmod \left(\exists(V-\{p\}) \cdot \varphi_{i}\right)$, and $\omega \in \bmod \left(\wedge_{i=1}^{n} \exists(V-\{p\}) \cdot \varphi_{i}\right)$. So it is consistent. If $\omega \models \neg l$, then there is a model $\omega^{\prime} \in \bmod \left(\wedge_{i=1}^{n} \exists V \cdot \varphi_{i}\right)$ satisfying $\omega^{\prime}$ and $\omega$ give the same truth value except $p$. It can be reduced to the first case and $\omega^{\prime} \in \bmod \left(\wedge_{i=1}^{n} \exists(V-\{p\}) \cdot \varphi_{i}\right)$. Thus $\wedge_{i=1}^{n} \exists(V-\{p\}) \cdot \varphi_{i}$ is also consistent.

The lemma indicates that the minimal forgetting set of variables doesn't comprise the variables on which all KBs agree. It can be easily extended to the general case that $p$ doesn't occur in some bases and the other bases including it entail $l$.

Theorem 3: $\triangle_{\mu}^{f_{1}}$ satisfies properties (A1), (A2).

Proof: (A1) Let $V^{\prime}=\operatorname{Var}(\Phi)-\{p\}$. If $\forall \varphi^{\prime} \in \Phi^{\prime}, \varphi^{\prime} \models p$, then $\forall \varphi^{\prime} \in \Phi^{\prime}, \exists V^{\prime} . \varphi^{\prime} \models p$. So $\exists V^{\prime} . \varphi^{\prime} \equiv p$. And $\forall \varphi \in$ $\Phi-\Phi^{\prime}, \exists V^{\prime} . \varphi \equiv \top$. Thus if $\mu \not \forall \neg p$ then $\wedge_{\varphi \in \Phi}\left(\exists V^{\prime} . \varphi\right) \wedge \mu \equiv$ $p \wedge \mu$ is consistent. In the definition of $\triangle_{\mu}^{f_{1}}, \forall V \in F S, p \notin$ $V$. Otherwise, $V-\{p\}$ is minimal variables set such that $\wedge_{\varphi \in \Phi}(\exists(V-\{p\}) \cdot \varphi) \wedge \mu$ is consistent. Thus $\triangle_{\mu}^{f_{1}}(\Phi) \models p$.

(A2) $\forall V \in F S, p \in V$ holds. Otherwise, suppose $p \notin V$. Let $\varphi_{1} \models p$ and $\varphi_{2} \models \neg p$. So $\exists V . \varphi_{1} \models p$ and $\exists V . \varphi_{2} \models$ $\neg p . \exists V . \varphi_{1} \wedge \exists V . \varphi_{2}$ is inconsistent. $V \notin F S$. The property is proved.

We return to consider the motivating example. $\varphi_{1}\left(\varphi_{2}\right)$ and $\varphi_{3}$ assign contrary truth values on variables $S, T, P$. So they should forget these variables to preserve consistency. $V=\{S, T, P\}$ is the only element in $F S$ of definition above, $\triangle_{\mu}^{f_{1}}(\Phi)=\wedge_{i=1}^{4} \exists V \cdot \varphi_{i} \wedge \mu=\neg I \wedge \mu=\neg I \wedge(\neg S \vee \neg T) \wedge$ $(\neg S \vee \neg P) \wedge(\neg P \vee \neg T)$.

Before we prepare to show properties of $\triangle_{\mu}^{f_{1}}$, we first introduce an important lemma.

Lemma 3: Let $\varphi, \varphi^{\prime}$ be two formulas and $V$ a set of variables. We have $d\left(\exists V . \varphi, \varphi^{\prime}\right)=d\left(\exists V . \varphi, \exists V . \varphi^{\prime}\right)$.

Proof: We prove first $d\left(\exists\{p\} . \varphi, \varphi^{\prime}\right)=$ $d\left(\exists\{p\} \cdot \varphi, \exists\{p\} \cdot \varphi^{\prime}\right)$ for any atom p. $\operatorname{Mod}\left(\varphi^{\prime}\right) \subseteq$ $\operatorname{Mod}\left(\exists\{p\} \cdot \varphi^{\prime}\right)$, so $d\left(\exists\{p\} \cdot \varphi, \exists\{p\} \cdot \varphi^{\prime}\right) \leq d\left(\exists\{p\} \cdot \varphi, \varphi^{\prime}\right)$. Take $\omega \in \operatorname{Mod}(\exists\{p\} \cdot \varphi), \omega^{\prime} \in \operatorname{Mod}\left(\exists\{p\} \cdot \varphi^{\prime}\right)$, $d\left(\omega, \omega^{\prime}\right)=d\left(\exists\{p\} . \varphi, \exists\{p\} . \varphi^{\prime}\right)$. There are four cases:

1. If $\omega \in \operatorname{Mod}(\varphi), \omega^{\prime} \in \operatorname{Mod}\left(\varphi^{\prime}\right)$, then the equation holds.

2. If $\omega \in \operatorname{Mod}(\varphi), \omega^{\prime} \in \operatorname{Mod}\left(\exists\{p\} . \varphi^{\prime}\right)-\operatorname{Mod}\left(\varphi^{\prime}\right)$, let $\omega_{1}, \omega_{1}^{\prime}$ assign differently only on $p$ with $\omega, \omega^{\prime}$ respectively. Then $d\left(\omega_{1}, \omega_{1}^{\prime}\right)=d\left(\omega, \omega^{\prime}\right)$ and $\omega_{1} \in \operatorname{Mod}(\exists\{p\} \cdot \varphi), \omega_{1}^{\prime} \in \operatorname{Mod}\left(\varphi^{\prime}\right)$. So the equation holds.

3. If $\omega \in \operatorname{Mod}(\exists\{p\} . \varphi)-\operatorname{Mod}(\varphi), \omega \in \operatorname{Mod}\left(\varphi^{\prime}\right)$, then the equation holds.

4. If $\omega \in \operatorname{Mod}(\exists\{p\} . \varphi)-\operatorname{Mod}(\varphi), \omega^{\prime} \in \operatorname{Mod}\left(\exists\{p\} . \varphi^{\prime}\right)-$ $\operatorname{Mod}\left(\varphi^{\prime}\right)$, Let $\omega_{1}, \omega_{1}^{\prime}$ assign differently only on $p$ with $\omega, \omega^{\prime}$ respectively. Then $d\left(\omega_{1}, \omega_{1}^{\prime}\right)=d\left(\omega, \omega^{\prime}\right)$ and $\omega_{1} \in \operatorname{Mod}(\varphi), \omega_{1}^{\prime} \in \operatorname{Mod}\left(\varphi^{\prime}\right)$. So the equation holds.

Next we prove the general case for any set $V(|V|>1)$. Let $V=\left\{p_{1}, \cdots, p_{n}\right\} . d\left(\exists V \cdot \varphi, \exists V \cdot \varphi^{\prime}\right)$ 
$=d\left(\exists\left\{p_{1}\right\} \cdot\left(\exists\left(V-\left\{p_{1}\right\}\right) \cdot \varphi\right), \exists\left\{p_{1}\right\} \cdot\left(\exists\left(V-\left\{p_{1}\right\}\right) \cdot \varphi^{\prime}\right)\right)$

$=d\left(\exists\left\{p_{1}\right\} \cdot\left(\exists\left(V-\left\{p_{1}\right\}\right) \cdot \varphi\right), \exists\left(V-\left\{p_{1}\right\}\right) \cdot \varphi^{\prime}\right)$

$=d\left(\exists V \cdot \varphi, \exists\left(V-\left\{p_{1}\right\}\right) \cdot \varphi^{\prime}\right)$

$=d\left(\exists V \cdot \varphi, \exists\left(V-\left\{p_{1}, p_{2}\right\}\right) \cdot \varphi^{\prime}\right)$

$=\cdots$

$=d\left(\exists V \cdot \varphi, \varphi^{\prime}\right)$.

This lemma shows $d\left(\exists V \cdot \varphi, \varphi^{\prime}\right)=d\left(\exists V \cdot \varphi^{\prime}, \varphi\right)$. So $\exists V \cdot \varphi$ is consistent with $\varphi^{\prime}$ iff $\exists V . \varphi^{\prime}$ is consistent with $\varphi$.

It's easy to see that for any $V^{\prime} \subseteq V, d\left(\exists V . \varphi, \varphi^{\prime}\right) \equiv$ $d\left(\exists V . \varphi, \exists V^{\prime} . \varphi^{\prime}\right)$. But $\exists V \cdot \varphi \wedge \varphi^{\prime} \equiv \exists V \cdot \varphi \wedge \exists V^{\prime} \cdot \varphi^{\prime}$ doesn’t hold. For example, $\varphi \equiv p \wedge q, \varphi^{\prime} \equiv \neg p . \exists\{p\} . \varphi \wedge \varphi^{\prime} \equiv q \wedge \neg p$, but $\exists\{p\} \cdot \varphi \wedge \exists\{p\} \cdot \varphi^{\prime} \equiv q$.

Theorem 4: $\triangle_{\mu}^{f_{1}}(\Phi)$ satisfies postulates (IC0)-(IC4), (IC7), (IC8) and (MI).

Proof: 1. (IC0)-(IC3) obviously hold for $\triangle_{\mu}^{f_{1}}(\Phi)$.

2. $\triangle_{\mu}^{f_{1}}\left(\varphi \cup \varphi^{\prime}\right) \wedge \varphi=\vee_{V \in F S}\left(\exists V . \varphi \wedge \exists V . \varphi^{\prime} \wedge \mu\right) \wedge \varphi=$ $\vee_{V \in F S}\left(\varphi \wedge \exists V \cdot \varphi^{\prime}\right)$ and $\triangle_{\mu}^{f_{1}}\left(\varphi \cup \varphi^{\prime}\right) \wedge \varphi^{\prime}=\vee_{V \in F S}\left(\varphi^{\prime} \wedge \exists V \cdot \varphi\right)$. According to lemma 3 above, $\exists V \cdot \varphi \wedge \exists V . \varphi^{\prime}$ is consistent if and only if $\exists V . \varphi \wedge \varphi^{\prime}$ (or $\exists V . \varphi^{\prime} \wedge \varphi$ ) is consistent. So (IC4) holds.

3. Now we prove (IC7) and (IC8). FS, F $S^{\prime}$ are collections of forgetting set for $\triangle_{\mu_{1}}(\Phi)$ and $\triangle_{\mu_{1} \wedge \mu_{2}}(\Phi)$ respectively in the definition. Let $\triangle_{\mu_{1}}^{f_{1}}(\Phi)=\vee_{V \in F S}\left(\wedge_{i=1}^{n} \exists V \cdot \varphi_{i} \wedge \mu_{1}\right)$. If $\triangle_{\mu_{1}}^{f_{1}}(\Phi) \wedge \mu_{2}$ is consistent, then $\exists V_{0}$ satisfying that $\wedge_{i=1}^{n} \exists V_{0} \cdot \varphi_{i} \wedge \mu_{1} \wedge \mu_{2}$ is consistent. The cardinals of sets in $F S, F S^{\prime}$ are the same. So $V_{0} \in F S^{\prime}$. Conversely, $\forall V^{\prime} \in F S^{\prime}$, $V^{\prime} \in F S$. Then we have $\triangle_{\mu_{1}}^{f_{1}}(\Phi) \wedge \mu_{2} \equiv \triangle_{\mu_{1} \wedge \mu_{2}}^{f_{1}}(\Phi)$.

4. For some set $V$, the conjunction of every formula in $\Phi_{1} \sqcup \Phi_{2}^{n}$ after forgetting $V$ is consistent if and only if it is the same case for $\Phi_{1} \sqcup \Phi_{2}$ after forgetting $V$. The collection of forgetting sets in $\triangle_{\mu}^{f_{1}}\left(\Phi_{1} \sqcup \Phi_{2}^{n}\right)$ is the same as the one for $\triangle_{\mu}^{f_{1}}\left(\Phi_{1} \sqcup \Phi_{2}\right)$. The knowledge set $\Phi_{1} \sqcup \Phi_{2}^{n}$ after forgetting $V \in F S$ is

$\vee_{V \in F S}\left(\wedge_{\varphi \in \Phi_{1}} \exists V \cdot \varphi \wedge_{i=1}^{n}\left(\wedge_{\psi \in \Phi_{2}} \exists V \cdot \psi\right) \wedge \mu\right)$

$=\vee_{V \in F S}\left(\wedge_{\varphi \in \Phi_{1}} \exists V \cdot \varphi \wedge_{\psi \in \Phi_{2}} \exists V \cdot \psi \wedge \mu\right)$. Thus after forgetting $V$, two knowledge sets $\Phi_{1} \sqcup \Phi_{2}^{n}$ and $\Phi_{1} \sqcup \Phi_{2}$ become identical, i.e., $\triangle_{\mu}^{f_{1}}\left(\Phi_{1} \sqcup \Phi_{2}^{n}\right) \equiv \triangle_{\mu}^{f_{1}}\left(\Phi_{1} \sqcup \Phi_{2}\right)$.

The operator $\triangle_{\mu}^{f_{1}}$ doesn't satisfy postulates (IC5) and (IC6), but it satisfies the proposed properties (A1) and (A2). We think that $\triangle_{\mu}\left(\Phi_{1}\right) \wedge \triangle_{\mu}\left(\Phi_{2}\right)$ doesn't represent the common alternatives of the two groups which are indeed $T h\left(\triangle_{\mu}\left(\Phi_{1}\right)\right) \cap T h\left(\triangle_{\mu}\left(\Phi_{2}\right)\right)(T h(\varphi)$ denotes the deduction closure of formula $\varphi$ ). If we select the minimal sets w.r.t setinclusion then we get another operator.

Definition 2: Let $\Phi=\left\{\varphi_{1}, \varphi_{2}, \cdots, \varphi_{n}\right\}$ and $\mu$ an integrity constraint. $V \subseteq \cup_{i=1}^{n} \operatorname{Var}\left(\varphi_{i}\right)$, the minimal number of variables for forgetting $F S=\{V$ is minimal w.r.t set-inclusion | $\wedge_{i=1}^{n} \exists V \cdot \varphi \wedge \mu$ is consistent $\}$. We define an operator as follows: $\triangle_{\mu}^{f_{2}}(\Phi)=\vee_{V \in F S}\left(\wedge_{i=1}^{n} \exists V . \varphi \wedge \mu\right)$.

For the example of co-owners, The results of $\triangle_{\mu}^{f_{2}}$ and $\triangle_{\mu}^{f_{1}}$ are the same one. Analogously, $\triangle_{\mu}^{f_{2}}$ satisfies (A1), (A2) and some postulates.

Theorem 5: $\triangle_{\mu}^{f_{2}}$ satisfies (A1), (A2), (IC0)-(IC4), (IC7) and (MI).

The next theorem states that the property (IC8) doesn't hold, thereby $\triangle_{\mu}^{f_{1}}$ and $\triangle_{\mu}^{f_{2}}$ are different. For example, Let $\Phi=$ $\left\{\varphi_{1}, \varphi_{2}\right\}, \mu=\top$ and $\varphi_{1}=\neg p \wedge \neg q \wedge \neg r \wedge \neg s, \varphi_{2}=((p \wedge$ $\neg q \wedge \neg r) \vee(\neg p \wedge q \wedge r)) \wedge \neg s .\{a\},\{b, c\}$ are the forgetting sets for $\triangle_{\mu}^{f_{2}}$, but $\{a\}$ is the only one for $\triangle_{\mu}^{f_{1}}$. So $\triangle_{\mu}^{f_{1}}(\Phi) \equiv$ $\neg q \wedge \neg r \wedge \neg s, \triangle_{\mu}^{f_{2}} \equiv \neg p \wedge \neg s$.

These two operators belong to homogeneous context in [10] in which they propose three contexts for forgetting.

\section{CONCLUSion ANd Future Work}

This paper proposes a scenario to eliminate conflicts occurring in the process of merging KBs by applying variable forgetting. Firstly, we discuss the relationship between belief merging and variable forgetting for KBs via the operation of dilation. As an interesting result, three classical model-based merging operators can be well captured by variable forgetting. Based on this relationship, we revise those merging operators by modifying variables in forgetting so that these new operators (after revising) become more smart in managing multiple KBs. Though our work is inspired from [10], our operator is based on variable selection on multiple KBs while [10] is based on the context of singe KB. Because propositional logic has limited power of expression, as a future work, we will consider our forgetting-based merging in a broad logic language such as description logic which is proved to be a highly successful class of knowledge representation languages in the Semantic Web.

\section{ACKNOWLEDGEMENTS}

This work is supported by NSFC under grant number 60973003, 60496322 and the Ph.D. Programs Foundation of Ministry of Education of China.

\section{REFERENCES}

[1] Liberatore, P. and Schaerf, M., Arbitration (or how to merge knowledge bases), IEEE Transactions on Knowledge and Data Engineering, vol. 10(1), pp. 76-90, 1998.

[2] Konieczny, S. and Pérez, R.P., Merging information under constraints: a logical framework, Journal of Logic and Computation, Oxford University Press, vol. 12, pp. 773-808, 2002.

[3] Gorogiannis, N. and Hunter, A., Merging first-order knowledge using dilation operators, in Proc. international conference on Foundations of information and knowledge systems (FoIKS'08), ser. Lecture Notes in Computer Science. Berlin, Germany: Springer, vol. 4932. pp. 132-150, 2008.

[4] Konieczny, S., Lang, J. and Marquis, P., DA2 merging operators, Artificial Intelligence, Elsevier, vol. 157(1-2), pp. 49-79, 2004.

[5] Dalal, M., Investigations into a theory of knowledge base revision: Preliminary report, in Proc. National Conference on Artificial Intelligence (AAAI'88), AAAI Press / The MIT Press, vol. 2, pp. 475-479, 1988.

[6] Lin, J. and Mendelzon, A.O., Knowledge base merging by majority, Dynamic Worlds: From the Frame Problem to Knowledge Management, pp. 195-218, 1999.

[7] Revesz, P.Z., On the semantics of theory change: arbitration between old and new information, in Proc. ACM SIGACT-SIGMOD-SIGART symposium on Principles of database systems (PODS'93), Washington, DC. ACM Press pp.71-82, 1993.

[8] Silberschatz, A., Stonebraker, M. and Ullman, J.D., Database systems: Achievements and opportunities, Communications of the ACM, Association for Computing Machinery, vol. 34(10), pp. 110-120, 1991.

[9] Lin,F. and Reiter,R., Forget It!, in Proc. AAAI Fall Symposium on Relevance, pp. 154-159, 1994.

[10] Lang, J. and Marquis, P., Reasoning under inconsistency: A forgettingbased approach, Artificial Intelligence, Elsevier, vol. 174, pp. 799C823, 2010 . 